\title{
COEFFICIENT ESTIMATES FOR NEW SUBCLASSES OF ANALYTIC FUNCTIONS WITH RESPECT TO OTHER POINTS
}

\author{
HUO TANG AND GUAN-TIE DENG
}

\begin{abstract}
The main purpose of this paper is to derive coefficient estimates for new subclasses of analytic functions with respect to symmetric and conjugate points.
\end{abstract}

\section{Introduction}

Let $U$ be the class of functions which are analytic and univalent in the open unit disk $D=\{z:|z|<1\}$ given by

$$
\omega(z)=\sum_{k=1}^{\infty} b_{k} z^{k}
$$

and satisfying the conditions $\omega(0)=0,|\omega(z)| \leq 1, z \in D$.

Let $S$ denote the class of functions $f$ which are analytic and univalent in $D$ of the form

$$
f(z)=z+\sum_{n=2}^{\infty} a_{n} z^{n}(z \in D)
$$

Let $S_{s}^{*}$ be the subclass of $S$ consisting of functions given by (1.2) and satisfying the condition

$$
\operatorname{Re}\left(\frac{z f^{\prime}(z)}{f(z)-f(-z)}\right)>0 \quad(z \in D) .
$$

These functions are called starlike with respect to symmetric points and were introduced by Sakaguchi [1].

Also, let $S_{c}^{*}$ be the subclass of $S$ consisting of functions given by (1.2) and satisfying the condition

$$
R e\left(\frac{z f^{\prime}(z)}{f(z)+\overline{f(\bar{z})}}\right)>0 \quad(z \in D) .
$$

These functions are called starlike with respect to conjugate points and were introduced by Ashwah and Thomas [2].

Corresponding author: Huo Tang.

2010 Mathematics Subject Classification. 30C45.

Key words and phrases. Analytic functions, symmetric, conjugate, coefficient estimates. 
Motivated by the class $S_{s}^{*}$, Das and Singh [3] discussed the following class $C_{s}$, namely convex functions with respect to symmetric points.

Let $C_{s}$ be the subclass of $S$ consisting of functions given by (1.2) satisfying the condition

$$
\operatorname{Re}\left(\frac{\left(z f^{\prime}(z)\right)^{\prime}}{(f(z)-f(-z))^{\prime}}\right)>0 \quad(z \in D) .
$$

Suppose that $f$ and $g$ are two analytic functions in $U$. Then, we say that the function $g$ is subordinate to the function $f$, and we write

$$
g(z) \prec f(z) \quad(z \in D)
$$

if there exists a Schwarz function $\varrho(z)$ with $\varrho(0)=0$ and $|\varrho(z)|<1$ such that

$$
g(z)=f(\varrho(z)) \quad(z \in D)
$$

By applying the above subordination definition, Goel and Mehrok [4] introduced a subclass of $S_{s}^{*}$ denoted by $S_{s}^{*}(A, B)$.

Let $S_{s}^{*}(A, B)$ be the class of functions of the form (1.2) and satisfying the condition

$$
\frac{2 z f^{\prime}(z)}{f(z)-f(-z)} \prec \frac{1+A z}{1+B z} \quad(-1 \leq B<A \leq 1 ; z \in D) .
$$

Also, in the same manner, we give the analogue definitions by extension as follows.

\section{Definition 1.1.}

(i) Let $S_{c}^{*}(A, B)$ be the subclass of $S$ consisting of functions given by (1.2) satisfying the condition

$$
\frac{2 z f^{\prime}(z)}{f(z)+\overline{f(\bar{z})}}<\frac{1+A z}{1+B z}(-1 \leq B<A \leq 1, z \in D) .
$$

(ii) Let $C_{S}(A, B)$ be the subclass of $S$ consisting of functions given by (1.2) satisfying the condition

$$
\frac{2\left(z f^{\prime}(z)\right)^{\prime}}{(f(z)-f(-z))^{\prime}}<\frac{1+A z}{1+B z}(-1 \leq B<A \leq 1, z \in D) .
$$

(iii) Let $C_{c}(A, B)$ be the subclass of $S$ consisting of functions given by (1.2) satisfying the condition

$$
\frac{2\left(z f^{\prime}(z)\right)^{\prime}}{(f(z)+\overline{f(\bar{z})})^{\prime}}<\frac{1+A z}{1+B z}(-1 \leq B<A \leq 1, z \in D) .
$$

In this paper, we introduce the class $M_{s}(\alpha, \mu, A, B)$ consisting of analytic functions $f$ of the form (1.2) and satisfying

$$
\frac{2 \alpha \mu z^{3} f^{\prime \prime \prime}(z)+2(2 \alpha \mu+\alpha-\mu) z^{2} f^{\prime \prime}(z)+2 z f^{\prime}(z)}{\alpha \mu z^{2}(f(z)-f(-z))^{\prime \prime}+(\alpha-\mu) z(f(z)-f(-z))^{\prime}+(1-\alpha+\mu)(f(z)-f(-z))}<\frac{1+A z}{1+B z},
$$


where $-1 \leq B<A \leq 1,0 \leq \mu \leq \alpha \leq 1$ and $z \in D$.

In addition, we introduce the class $M_{c}(\alpha, \mu, A, B)$ consisting of analytic functions $f$ of the form (1.2) and satisfying

$$
\frac{2 \alpha \mu z^{3} f^{\prime \prime \prime}(z)+2(2 \alpha \mu+\alpha-\mu) z^{2} f^{\prime \prime}(z)+2 z f^{\prime}(z)}{\alpha \mu z^{2}(f(z)+\overline{f(\bar{z})})^{\prime \prime}+(\alpha-\mu) z(f(z)+\overline{f(\bar{z})})^{\prime}+(1-\alpha+\mu)(f(z)+\overline{f(\bar{z})})}<\frac{1+A z}{1+B z},
$$

where $-1 \leq B<A \leq 1,0 \leq \mu \leq \alpha \leq 1$ and $z \in D$.

We note that

(i) for $\mu=0, M_{s}(\alpha, 0, A, B)=M_{s}(\alpha, A, B)$ and $M_{c}(\alpha, 0, A, B)=M_{c}(\alpha, A, B)$, which were introduced and studied by Selvaraj and Vasanthi [5];

(ii) for $\mu=\alpha=0, M_{s}(0,0, A, B)=S_{s}^{*}(A, B)$ and $M_{c}(0,0, A, B)=S_{c}^{*}(A, B)$;

(iii) for $\mu=0$ and $\alpha=1, M_{s}(1,0, A, B)=C_{s}(A, B)$ and $M_{c}(1,0, A, B)=C_{c}(A, B)$.

By the definition of subordination, it follows that $f \in M_{s}(\alpha, \mu, A, B)$ if and only if

$$
\begin{aligned}
& \frac{2 \alpha \mu z^{3} f^{\prime \prime \prime}(z)+2(2 \alpha \mu+\alpha-\mu) z^{2} f^{\prime \prime}(z)+2 z f^{\prime}(z)}{\alpha \mu z^{2}(f(z)-f(-z))^{\prime \prime}+(\alpha-\mu) z(f(z)-f(-z))^{\prime}+(1-\alpha+\mu)(f(z)-f(-z))} \\
& \quad=\frac{1+A \omega(z)}{1+B \omega(z)}=p(z), \omega(z) \in U
\end{aligned}
$$

and that $f \in M_{c}(\alpha, \mu, A, B)$ if and only if

$$
\begin{aligned}
& \frac{2 \alpha \mu z^{3} f^{\prime \prime \prime}(z)+2(2 \alpha \mu+\alpha-\mu) z^{2} f^{\prime \prime}(z)+2 z f^{\prime}(z)}{\alpha \mu z^{2}(f(z)+\overline{f(\bar{z})})^{\prime \prime}+(\alpha-\mu) z(f(z)+\overline{f(\bar{z})})^{\prime}+(1-\alpha+\mu)(f(z)+\overline{f(\bar{z})})} \\
& =\frac{1+A \omega(z)}{1+B \omega(z)}=p(z), \quad \omega(z) \in U
\end{aligned}
$$

where

$$
p(z)=1+\sum_{n=1}^{\infty} p_{n} z^{n}
$$

In the next section, we obtain the coefficient estimates for functions belonging to the classes $M_{s}(\alpha, \mu, A, B)$ and $M_{c}(\alpha, \mu, A, B)$.

\section{Main results}

In order to prove our main results, we shall require the following lemma due to Goel and Mehrok [4].

Lemma 2.1. If $p(z)$ is given by (1.7), then

$$
\left|p_{n}\right| \leq(A-B), \quad n=1,2, \ldots
$$


Unless otherwise mentioned, we shall assume in the reminder of this paper that $-1 \leq B<$ $A \leq 1,0 \leq \mu \leq \alpha \leq 1$ and $z \in D$.

Theorem 2.1. Let $f \in M_{s}(\alpha, \mu, A, B)$. Then, for $n \geq 1$, we have

$$
\begin{aligned}
\left|a_{2 n}\right| & \leq \frac{(A-B)}{2^{n} \cdot n ![1+(2 n-1)(\alpha-\mu+2 n \alpha \mu)]} \prod_{j=1}^{n-1}(A-B+2 j), \\
\left|a_{2 n+1}\right| & \leq \frac{(A-B)}{2^{n} \cdot n ![1+2 n(\alpha-\mu+(2 n+1) \alpha \mu)]} \prod_{j=1}^{n-1}(A-B+2 j) .
\end{aligned}
$$

Proof. From (1.5) and (1.7), we have

$$
\begin{aligned}
{[z+2} & \left.a_{2} z^{2}+3 a_{3} z^{3}+4 a_{4} z^{4}+5 a_{5} z^{5}+\cdots+2 n a_{2 n} z^{2 n}+\cdots\right] \\
& +(2 \alpha \mu+\alpha-\mu)\left[2 a_{2} z^{2}+6 a_{3} z^{3}+12 a_{4} z^{4}+20 a_{5} z^{5}+\cdots+(2 n-1) 2 n a_{2 n} z^{2 n}+\cdots\right] \\
& +\alpha \mu\left[6 a_{3} z^{3}+24 a_{4} z^{4}+60 a_{5} z^{5}+\cdots+(2 n-1) 2 n(2 n+1) a_{2 n+1} z^{2 n+1}+\cdots\right] \\
= & {\left[(1+\alpha-\mu)\left[z+a_{3} z^{3}+a_{5} z^{5}+\cdots+a_{2 n-1} z^{2 n-1}+a_{2 n+1} z^{2 n+1}+\cdots\right]\right.} \\
& +(\alpha-\mu)\left[z+3 a_{3} z^{3}+5 a_{5} z^{5}+\cdots+(2 n-1) a_{2 n-1} z^{2 n-1}+(2 n+1) a_{2 n+1} z^{2 n+1}+\cdots\right] \\
& \left.+\alpha \mu\left[6 a_{3} z^{3}+20 a_{5} z^{5}+\cdots+2 n(2 n+1) a_{2 n+1} z^{2 n+1}+\cdots\right]\right] \\
& \cdot\left[1+p_{1} z+p_{2} z^{2}+p_{3} z^{3}+p_{4} z^{4}+p_{5} z^{5}+\cdots+p_{2 n-1} z^{2 n-1}+p_{2 n} z^{2 n}+\cdots\right] .
\end{aligned}
$$

Equating the coefficients of like powers of $z$, we obtain

$$
\begin{aligned}
2[1+(\alpha-\mu+2 \alpha \mu)] a_{2} & =p_{1}, \quad 2[1+2(\alpha-\mu+3 \alpha \mu)] a_{3}=p_{2} \\
4[1+3(\alpha-\mu+4 \alpha \mu)] a_{4}= & p_{3}+[1+2(\alpha-\mu+3 \alpha \mu)] a_{3} p_{1} \\
4[1+4(\alpha-\mu+5 \alpha \mu)] a_{5}= & p_{4}+[1+2(\alpha-\mu+3 \alpha \mu)] a_{3} p_{2} \\
2 n[1+(2 n-1)(\alpha-\mu+2 n \alpha \mu)] a_{2 n}= & p_{2 n-1}+[1+2(\alpha-\mu+3 \alpha \mu)] a_{3} p_{2 n-3}+\cdots \\
& +[1+(2 n-2)(\alpha-\mu+(2 n-1) \alpha \mu)] a_{2 n-1} p_{1} \\
(2 n+1)[1+2 n(\alpha-\mu+(2 n+1) \alpha \mu)] a_{2 n+1}= & p_{2 n}+[1+2(\alpha-\mu+3 \alpha \mu)] a_{3} p_{2 n-2}+\cdots \\
& +[1+(2 n-2)(\alpha-\mu+(2 n-1) \alpha \mu)] a_{2 n-1} p_{2}
\end{aligned}
$$

By using Lemma 2.1 and (2.4), we get

$$
\left|a_{2}\right| \leq \frac{A-B}{2[1+(\alpha-\mu+2 \alpha \mu)]}, \quad\left|a_{3}\right| \leq \frac{A-B}{2[1+2(\alpha-\mu+3 \alpha \mu)]} .
$$

Again, making use of (2.1), in conjunction with (2.9), we find from (2.5) and (2.6) that

$$
\left|a_{4}\right| \leq \frac{(A-B)(A-B+2)}{2 \cdot 4 \cdot[1+3(\alpha-\mu+4 \alpha \mu)]},
$$




$$
\left|a_{5}\right| \leq \frac{(A-B)(A-B+2)}{2 \cdot 4 \cdot[1+4(\alpha-\mu+5 \alpha \mu)]} .
$$

It follows that (2.2) and (2.3) hold for $n=1,2$. Next, we prove (2.2) by induction.

Equation (2.7) together with Lemma 2.1 yields

$$
\left|a_{2 n}\right| \leq \frac{(A-B)}{2 n[1+(2 n-1)(\alpha-\mu+2 n \alpha \mu)]}\left\{1+\sum_{k=1}^{n-1}[1+2 k(\alpha-\mu+(2 k+1) \alpha \mu)]\left|a_{2 k+1}\right|\right\} .
$$

We suppose that (2.2) holds for $k=3,4, \ldots,(n-1)$.

Then from (2.10), we have

$$
\left|a_{2 n}\right| \leq \frac{(A-B)}{2 n[1+(2 n-1)(\alpha-\mu+2 n \alpha \mu)]}\left[1+\sum_{k=1}^{n-1} \frac{A-B}{2^{k} k !} \prod_{j=1}^{k-1}(A-B+2 j)\right] .
$$

In order to complete the proof, it is sufficient to show that

$$
\begin{aligned}
& \frac{(A-B)}{2 m[1+(2 m-1)(\alpha-\mu+2 m \alpha \mu)]}\left[1+\sum_{k=1}^{m-1} \frac{A-B}{2^{k} k !} \prod_{j=1}^{k-1}(A-B+2 j)\right] \\
& =\frac{(A-B)}{2^{m} \cdot m ![1+(2 m-1)(\alpha-\mu+2 m \alpha \mu)]} \prod_{j=1}^{m-1}(A-B+2 j) \quad(m=3,4, \ldots, n),
\end{aligned}
$$

which is valid for $m=3$.

Let us assume that (2.12) is true for all $m, 3<m \leq(n-1)$. Then from (2.11), we get

$$
\begin{aligned}
& \frac{(A-B)}{2 n[1+(2 n-1)(\alpha-\mu+2 n \alpha \mu)]}\left[1+\sum_{k=1}^{n-1} \frac{A-B}{2^{k} k !} \prod_{j=1}^{k-1}(A-B+2 j)\right] \\
&=\left(\frac{n-1}{n}\right)\left(\frac{(A-B)}{2(n-1)[1+(2 n-1)(\alpha-\mu+2 n \alpha \mu)]}\left(1+\sum_{k=1}^{n-2} \frac{(A-B)}{2^{k} k !} \prod_{j=1}^{k-1}(A-B+2 j)\right)\right) \\
&+\frac{(A-B)}{2 n[1+(2 n-1)(\alpha-\mu+2 n \alpha \mu)]} \cdot \frac{(A-B)}{2^{n-1} \cdot(n-1) !} \prod_{j=1}^{n-2}(A-B+2 j) \\
&=\left.\frac{n-1}{n}\right) \frac{(A-B)}{2^{n-1} \cdot(n-1) ![1+(2 n-1)(\alpha-\mu+2 n \alpha \mu)]} \prod_{j=1}^{n-2}(A-B+2 j) \\
&+\frac{(A-B)}{2 n[1+(2 n-1)(\alpha-\mu+2 n \alpha \mu)]} \cdot \frac{(A-B)}{2^{n-1} \cdot(n-1) !} \prod_{j=1}^{n-2}(A-B+2 j) \\
&= \frac{(A-B)}{2 n(n-1) ! 2^{n-1}[1+(2 n-1)(\alpha-\mu+2 n \alpha \mu)]} \prod_{j=1}^{n-2}(A-B+2 j)(A-B+2(n-1)) \\
&= \frac{(A-B)}{2^{n} \cdot n ![1+(2 n-1)(\alpha-\mu+2 n \alpha \mu)]} \prod_{j=1}^{n-1}(A-B+2 j) .
\end{aligned}
$$

Thus (2.12) holds for $m=n$ and hence (2.2) follows. Similarly, we can prove (2.3). 
Theorem 2.2. Let $f \in M_{c}(\alpha, \mu, A, B)$. Then, for $n \geq 1$, we have

$$
\begin{aligned}
\left|a_{2 n}\right| & \leq \frac{(A-B)}{(2 n-1) ![1+(2 n-1)(\alpha-\mu+2 n \alpha \mu)]} \prod_{j=1}^{2 n-2}(A-B+j), \\
\left|a_{2 n+1}\right| & \leq \frac{(A-B)}{(2 n) ![1+2 n(\alpha-\mu+(2 n+1) \alpha \mu)]} \prod_{j=1}^{2 n-1}(A-B+j) .
\end{aligned}
$$

Proof. From (1.6) and (1.7), we have

$$
\begin{aligned}
{[z+2} & \left.a_{2} z^{2}+3 a_{3} z^{3}+4 a_{4} z^{4}+5 a_{5} z^{5}+\cdots+2 n a_{2 n} z^{2 n}+\cdots\right] \\
& +(2 \alpha \mu+\alpha-\mu)\left[2 a_{2} z^{2}+6 a_{3} z^{3}+12 a_{4} z^{4}+20 a_{5} z^{5}+\cdots+(2 n-1) 2 n a_{2 n} z^{2 n}+\cdots\right] \\
& +\alpha \mu\left[6 a_{3} z^{3}+24 a_{4} z^{4}+60 a_{5} z^{5}+\cdots+(2 n-1) 2 n(2 n+1) a_{2 n+1} z^{2 n+1}+\cdots\right] \\
= & {\left[(1+\alpha-\mu)\left[z+a_{2} z^{2}+a_{3} z^{3}+a_{4} z^{4}+a_{5} z^{5}+\cdots+a_{2 n} z^{2 n}+\cdots\right]\right.} \\
& +(\alpha-\mu)\left[z+2 a_{2} z^{2}+3 a_{3} z^{3}+4 a_{4} z^{4}+5 a_{5} z^{5}+\cdots+2 n a_{2 n} z^{2 n}+\cdots\right] \\
& \left.+\alpha \mu\left[2 a_{2} z^{2}+6 a_{3} z^{3}+12 a_{4} z^{4}+20 a_{5} z^{5}+\cdots+(2 n-1) 2 n a_{2 n} z^{2 n}+\cdots\right]\right] \\
& \cdot\left[1+p_{1} z+p_{2} z^{2}+p_{3} z^{3}+p_{4} z^{4}+p_{5} z^{5}+\cdots+p_{2 n-1} z^{2 n-1}+\cdots\right]
\end{aligned}
$$

Equating the coefficients of like powers of $z$, we obtain

$$
\begin{aligned}
& {[1+(\alpha-\mu+2 \alpha \mu)] a_{2}=p_{1}, \quad 2[1+2(\alpha-\mu+3 \alpha \mu)] a_{3}=p_{2}+[1+(\alpha-\mu+2 \alpha \mu)] a_{2} p_{1} } \\
& 3[1+3(\alpha-\mu+4 \alpha \mu)] a_{4}=p_{3}+[1+(\alpha-\mu+2 \alpha \mu)] a_{2} p_{2}+[1+2(\alpha-\mu+3 \alpha \mu)] a_{3} p_{1} \\
& 4[1+4(\alpha-\mu+5 \alpha \mu)] a_{5}=p_{4}+[1+(\alpha-\mu+2 \alpha \mu)] a_{2} p_{3}+[1+2(\alpha-\mu+3 \alpha \mu)] a_{3} p_{2} \\
&+[1+3(\alpha-\mu+4 \alpha \mu)] a_{4} p_{1} \\
&(2 n-1)[1+(2 n-1)(\alpha-\mu+2 n \alpha \mu)] a_{2 n}= p_{2 n-1}+[1+(\alpha-\mu+2 \alpha \mu)] a_{2} p_{2 n-2}+\cdots \\
&+[1+(2 n-2)(\alpha-\mu+(2 n-1) \alpha \mu)] a_{2 n-1} p_{1} \\
&= p_{2 n}+[1+(\alpha-\mu+2 \alpha \mu)] a_{2} p_{2 n-1}+\cdots \\
&+[1+(2 n-1)(\alpha-\mu+2 n \alpha \mu)] a_{2 n} p_{1}
\end{aligned}
$$

By using Lemma 2.1 and (2.15), we obtain

$$
\left|a_{2}\right| \leq \frac{A-B}{[1+(\alpha-\mu+2 \alpha \mu)]}, \quad\left|a_{3}\right| \leq \frac{(A-B)(A-B+1)}{2[1+2(\alpha-\mu+3 \alpha \mu)]} .
$$

Again, making use of (2.1), in conjunction with (2.20), we find from (2.16) and (2.17) that

$$
\begin{aligned}
& \left|a_{4}\right| \leq \frac{(A-B)(A-B+1)(A-B+2)}{2 \cdot 3 \cdot[1+3(\alpha-\mu+4 \alpha \mu)]}, \\
& \left|a_{5}\right| \leq \frac{(A-B)(A-B+1)(A-B+2)(A-B+3)}{2 \cdot 3 \cdot 4 \cdot[1+4(\alpha-\mu+5 \alpha \mu)]} .
\end{aligned}
$$


It follows that (2.13) and (2.14) hold for $n=1,2$. Next, we prove (2.13) by induction.

Equation (2.18) together with Lemma 2.1 yields

$$
\begin{aligned}
\left|a_{2 n}\right| \leq & \frac{(A-B)}{(2 n-1)[1+(2 n-1)(\alpha-\mu+2 n \alpha \mu)]}\left\{1+\sum_{k=1}^{n-1}[1+(2 k-1)(\alpha-\mu+2 k \alpha \mu)]\left|a_{2 k}\right|\right. \\
& \left.+\sum_{k=1}^{n-1}[1+2 k(\alpha-\mu+(2 k+1) \alpha \mu)]\left|a_{2 k+1}\right|\right\} .
\end{aligned}
$$

We suppose that (2.13) holds for $k=3,4, \ldots,(n-1)$.

Then from (2.21), we obtain

$$
\begin{aligned}
\left|a_{2 n}\right| \leq & \frac{(A-B)}{(2 n-1)[1+(2 n-1)(\alpha-\mu+2 n \alpha \mu)]}\left[1+\sum_{k=1}^{n-1} \frac{(A-B)}{(2 k-1) !} \prod_{j=1}^{2 k-2}(A-B+j)\right. \\
& \left.+\sum_{k=1}^{n-1} \frac{(A-B)}{(2 k) !} \prod_{j=1}^{2 k-1}(A-B+j)\right] .
\end{aligned}
$$

In order to complete the proof, it is sufficient to show that

$$
\begin{aligned}
& \frac{(A-B)}{(2 m-1)[1+(2 m-1)(\alpha-\mu+2 m \alpha \mu)]} \\
& \quad \cdot\left[1+\sum_{k=1}^{m-1} \frac{(A-B)}{(2 k-1) !} \prod_{j=1}^{2 k-2}(A-B+j)+\sum_{k=1}^{m-1} \frac{(A-B)}{(2 k) !} \prod_{j=1}^{2 k-1}(A-B+j)\right] \\
& =\frac{(A-B)}{(2 m-1) ![1+(2 m-1)(\alpha-\mu+2 m \alpha \mu)]} \prod_{j=1}^{2 m-2}(A-B+j) \quad(m=3,4, \ldots, n),
\end{aligned}
$$

which is valid for $m=3$.

Let us assume that (2.23) is true for all $m, 3<m \leq(n-1)$. Then from (2.22), we get

$$
\begin{aligned}
& \frac{(A-B)}{(2 n-1)[1+(2 n-1)(\alpha-\mu+2 n \alpha \mu)]} \\
& \cdot\left[1+\sum_{k=1}^{n-1} \frac{(A-B)}{(2 k-1) !} \prod_{j=1}^{2 k-2}(A-B+j)+\sum_{k=1}^{n-1} \frac{(A-B)}{(2 k) !} \prod_{j=1}^{2 k-1}(A-B+j)\right] \\
& =\left(\frac{2 n-3}{2 n-1}\right)\left[\frac{(A-B)}{(2(n-1)-1)[1+(2 n-1)(\alpha-\mu+2 n \alpha \mu)]}\right. \\
& \left.\quad \cdot\left(1+\sum_{k=1}^{n-2} \frac{(A-B)}{(2 k-1) !} \prod_{j=1}^{2 k-2}(A-B+j)+\sum_{k=1}^{n-2} \frac{(A-B)}{(2 k) !} \prod_{j=1}^{2 k-1}(A-B+j)\right)\right] \\
& +\frac{(A-B)}{(2 n-1)[1+(2 n-1)(\alpha-\mu+2 n \alpha \mu)]} \cdot \frac{(A-B)}{(2(n-1)-1) !} \prod_{j=1}^{2 n-4}(A-B+j) \\
& +\frac{(A-B)}{(2 n-1)[1+(2 n-1)(\alpha-\mu+2 n \alpha \mu)]} \cdot \frac{(A-B)}{(2(n-1)) !} \prod_{j=1}^{2 n-3}(A-B+j)
\end{aligned}
$$




$$
\begin{aligned}
= & \left(\frac{2 n-3}{2 n-1}\right) \frac{(A-B)}{(2(n-1)-1) ![1+(2 n-1)(\alpha-\mu+2 n \alpha \mu)]} \prod_{j=1}^{2 n-4}(A-B+j) \\
& +\frac{(A-B)}{(2 n-1)[1+(2 n-1)(\alpha-\mu+2 n \alpha \mu)]} \cdot \frac{(A-B)}{(2(n-1)-1) !} \prod_{j=1}^{2 n-4}(A-B+j) \\
& +\frac{(A-B)}{(2 n-1)[1+(2 n-1)(\alpha-\mu+2 n \alpha \mu)]} \cdot \frac{(A-B)}{(2(n-1)) !} \prod_{j=1}^{2 n-3}(A-B+j) \\
= & \frac{(A-B)}{(2 n-1)(2(n-1)-1) ![1+(2 n-1)(\alpha-\mu+2 n \alpha \mu)]} \prod_{j=1}^{2 n-4}(A-B+j)(A-B+2 n-3) \\
& +\frac{(A-B)}{(2 n-1)[1+(2 n-1)(\alpha-\mu+2 n \alpha \mu)]} \cdot \frac{(A-B)}{(2(n-1)) !} \prod_{j=1}^{2 n-3}(A-B+j) \\
= & \frac{(A-B)}{(2 n-1) ![1+(2 n-1)(\alpha-\mu+2 n \alpha \mu)]} \prod_{j=1}^{2 n-2}(A-B+j) .
\end{aligned}
$$

Thus (2.23) holds for $m=n$ and hence (2.13) follows. Similarly, we can prove (2.14).

Remark 2.1. Taking $\mu=0$ in Theorems 2.1 and 2.2, we obtain the results obtained by Selvaraj and Vasanthi [5, Theorems 3.1 and 3.2, respectively].

\section{Acknowledgements}

The present investigation was partly supported by the Natural Science Foundation of China under Grant 11071020, the Higher School Doctoral Foundation of China under Grant 20100003110004 and the Natural Science Foundation of Inner Mongolia under Grant $2010 \mathrm{MS} 0117$.

\section{References}

[1] K. Sakaguchi, On certain univalent mappings, J. Math. Soc. Japan., 11 (1959), 72-75.

[2] R. M. El-Ashwah and D. K. Thomas, Some subclasses of close-to-convex functions, J. Ramanujan Math. Soc., 2 (1987), 86-100.

[3] R. N. Das and P. Singh, On subclasses of schlicht mapping, Indian J. Pure Appl. Math., 8 (1977), 864-872.

[4] R. M. Goel and B. C. Mehrok, A subclass of starlike functions with respect to symmetric points, Tamkang J. Math., 13 (1) (1982), 11-24.

[5] C. Selvaraj and N. Vasanthi, Subclasses of analytic functions with respect to symmetric and conjugate points, Tamkang J. Math., 42 (1) (2011), 87-94.

School of Mathematical Sciences, Beijing Normal University, Beijing 100875, China; School of Mathematics and Statistics, Chifeng University, Chifeng 024000, Inner Mongolia, China.

E-mail: thth2009@tom.com

School of Mathematical Sciences, Beijing Normal University, Beijing 100875, China.

E-mail: denggt@bnu.edu.cn 